\title{
AFRONTANDO LA POSIBILIDAD DE TENER CANCER DE MAMA*
}

\author{
CONFRONTING THE POSSIBILITY OF HAVING BREAST CANCER
}

\author{
BERTA VARELA**, BETTINA CAPORALE***, LUCIA DELGAD $0 * * * *$, \\ MERCEDESVIERA*****, ANA GALAIN $* * * * * *$ y LAURA SCHWARTZMAN N $* * * * * * *$
}

\begin{abstract}
RESUMEN
El impacto psicológico que implica recibir el Ilamado para una segunda prueba confirmatoria luego de la realización de la mamografía anual ha sido considerado en la literatura como un evento amenazante, desatando esfuerzos de afrontamiento. El presentetrabajo pretende conocer como afrontan las mujeres el segundo Ilamado y la respuesta emocional. Este estudio descriptivo observacional, utilizando métodos cuali y cuantitativos, incluyó 377 pacientes. Los instrumentos cuantitativos aplicados fueron la escala de depresión y ansi edad hospitalaria (HADS) y Perfil de estados deánimo (POM S). Se aplicó el M anual ULM como evaluación cualitativa del afrontamiento. Fueron estudiadas 377 mujeres, 37 (10\%) recibieron finalmente un diagnóstico de cáncer de mama. Estas pacientes reportaron 125 de los eventos de afrontamiento (mediana, 4 rango 1-13), mientras que las sanas reportaron 871 (mediana2, rango 0-9), diferencia estadísticamente significativa ( $M$ ann-Whitney test - two tailed: $p<0.0001$ ). Las pacientes fueron agrupadas en tres grupos de acuerdo a la clasificación dela mamografía G1 ( $\mathrm{n}$ : 279) para BI-RADS 1, 2 y 3; G2 (n: 48) síntomas clínicos y G3 (n:30) para BI-RADS 4 y 5. Las pacientes G3 mostraron un mayor uso del cluster, resignación como modo de afrontamiento $(p<0.001)$ y un menor uso del cluster aceptación $(\mathrm{p}<0.003)$. El $49 \%$ delas pacientes con cáncer utilizaron la resignación como afrontamiento, comparado con el $30 \%$ de las sanas $(p<0.04)$. $51 \%$ de las pacientes sanas utilizaron la aceptación, y $24 \%$ de las pacientes con cáncer $(p<0.003)$. La evitación y la desesperanza aumentan con la mayor probabilidad de tener cáncer. El afrontamiento diferenció a los grupos más claramente que los estados emocionales.
\end{abstract}

Palabras claves: Afrontamiento, screening, cáncer de mama, ansiedad, temor al cáncer.

\section{ABSTRACT}

The psychological impact of being recalled for a second-stage of breast cancer screening has been considered in the literature as a threatening event, triggering coping efforts. The present work focuses on the psychological cost of being recalled for further investigation after breast cancer screening tests. The descriptive observational study, using qual itative and quantitative methodologies included 377 patients. Quantitative applied instruments were Hospital Anxiety and Depression Scale (HADS) and Profile of M ood States (POM S). ULM M anual was applied as a qualitative evaluation of coping. $37(10 \%)$ who finally received a positive diagnosis of breast cancer. Cancer patients reported 125 of all coping events (median 4, range 1-13), while non-cancer patients reported

* Investigación financiada por la Comisión Honoraria de la Lucha contra el Cáncer, de U ruguay.

*** Licenciada en Psicología. Departamento de Psicología M édica. Hospital de Clínicas. Facultad de M edicina. M ontevideo, Uruguay. E-mail: bvarela@he.ed.uy

*** Licenciada en Psicología, asistente Departamento Psicología M édica, Facultad deM edicina, Universidad de La República. Uruguay. E-mail: caporaleb@yahoo.com

**** Profesora Agregada del Servicio de Oncologia Clínica y coordinadora de la Unidad Oncogenética del Hospital de Clínicas. Uruguay. E-mail: Idelgado@edu.uy

***** Licenciada en Psicología, asistente Departamento Psicología M édica, Facultad de M edicina, Universidad de La República. Uruguay. E-mail: merceva@adinet.com.uy

****** Licenciada en Psicología, asistente Departamento Psicología M édica, Facultad de M edicina, Universidad de La República. Uruguay. E-mail: agalain@adinet.com.uy

******* M édico psiquiatra. Profesora Agregada de Psicología M édica de la Facultad de M edicina, Universidad de la República Oriental del Uruguay. M iembro del Consejo Directivo de la International Society of Life Research (ISOQOL). Correspondencia: Calabria 3969, Montevideo, CP 11600, Uruguay. E-mail: lauras@mednet.org.uy 
871 (median 2, range 0-9), this difference being statistically significant (Mann-Whitney test - two tailed: $p<$ 0.0001). Patients were divided in three groups: $G 1$ ( $n: 279)$ for BI-RADS 1,2 and $3 ; G 2$ ( $n: 48)$ with presence of clinical symptoms and G3 ( $\mathrm{n}: 30$ ) for BI-RADS 4 and $5 . \mathrm{G} 3$ patients showed a higher use of resignation as coping pattern $(p<0.001)$ and a lower use of compliance $(p<0.003)$. Resignation pattern was used by $49 \%$ of those patients who finally received a positive diagnosis, compared to $30 \%$ of women who did not $(p<0.04) .51 \%$ of non-cancer patients used compliance, while only $24 \%$ of cancer patients did so $(p<0.003)$. Avoidance and hopelessness-hel plessness increase with cancer risk.

Keywords: Coping, breast cancer screening, anxiety, cancer fear.

Fecha recepción:16/04/07. Fecha aceptación: 29/10/07.

\section{INTRODUCCION}

La realización del examen de screening para detectar el cáncer de mama es una efectiva conducta de reducción de riesgo ya que puede incidir positivamente sobre el pronóstico delas pacientes a través deuna detección precoz. Stratman et al., (2004) encontraron que el diagnóstico precoz puede reducir el riesgo de muerte precoz del $79 \%$ al $56 \%$ a la edad de 50 años, mientras que a la edad de 79 años la relación es de 23 a $13 \%$, a la vez que otros estudios confirman estos hallazgos con una mayor posibilidad de reducir el tamaño del tumor en las primeras etapas ( $M$ ichaelson et al., 2003). Otros autores establecen una reducción del $63 \%$ en las muertes (Tabár, 2001) y la posibilidad de mejorar el pronóstico en mujeres que realizaron mamografías regulares entrelos 42 y 49 años (Buseman et al., 2003).

Es por ello que las mujeres deberían ser firmemente estimuladas para realizar estos exámenes. En relación con esta afirmación, algunos estudios (Bjurstam, Björneld \& Warwick, 2003; Duffy, Tabár \& Chen, 2002) muestran el impacto positivo que la organización de programas y servicios de screening tiene sobrela reducción de la mortalidad por cáncer de mama.

Los datos internacionales muestran que la prevalenciadeconductas descreening paracáncer de mama es actualmente baja. Blanchard et al. (2004) encontraron que sólo el $6 \%$ de las mujeres que se realizaron una mamografía la repitieron en los siguientes 10 años, y el promedio de mamografías recibidas en ese pe- riodo representó el $51 \%$ de la cifra recomendada por la Sociedad Americana de Cáncer. La mayor prevalencia de mamografías de screening se encontró en mujeres de entre 55 y 65 años y aquellas con antecedentes personales de cáncer de mama. Audrian et al. (1999) encontraron, en una muestra de 510 mujeres, que el $44 \%$ no realizaba el autoexamen, $29 \%$ lo realizaba oportunamentemostrando adhesión a esta conducta, 17\% lo sobre realizaba y el $10 \%$ lo realizaba en exceso.

Es de enorme importancia conocer cuáles son los factores que determinan la realización de exámenes de screening, ya que este conocimiento puede redundar en intervenciones dirigidas a promover esta conducta.

La preocupación por contraer cáncer de mama seencontró correlacionada con laspercepciones sobreel riesgo absoluto (riesgo personal) y riesgo comparativo (riesgo comparado con otras mujeres) (Lipkus, Halabi, Strigo \& Rimer, 2000). La pregunta a responder es si estas preocupaciones promueven realmente conductas de screening.

Las características sociodemográficas pueden estar relacionadas con loscomportamientos de screening. Banks et al. (2002) encontraron que las mujeres provenientes de áreas menos carenciadas eran más proclives a concurrir a estos programas que aquellas con un nivel socioeconómico inferior.

Los exámenes de screening tienen un gran impacto sobre las mujeres, siendo su susceptibilidad percibida (SP) a contraer cáncer fundamental para dicho impacto. El distrés causado por el screening parece ser mayor en 
mujeres con alta SP, y aún se mantiene durante un mes luego de recibir mamografías normales (Absetz, Aro \& Sutton, 2003).

Como sucede con otros exámenes descreening, existe un alto porcentaje de mamografías que arrojan resultados falso-positivos; es por ello que muchas mujeres son llamadas para realizar nuevos exámenes con el objetivo de confirmar o descartar el diagnóstico de cáncer. Esto debe obviamentetener un fuerte impacto sobre su estado emocional y cognitivo (Lampic, Thurfjell, Bergh \& Sjödén, 2001), ya que las pacientes deben enfrentar la incertidumbre de padecer cáncer y las consecuencias que esto implicaría (ej. cirugía, quimioterapia, radioterapia).

En relación con las mujeres que son nue vamentellamadas para repetir o completar sus exámenes luego de una mamografía patológica, el fuerte impacto psicológico de este evento sugiere la necesidad de disminuir el periodo de tiempo entre el llamado y el diagnóstico final, así como la necesidad de informar correctamente a las pacientes acerca de la existencia de un amplio rango de casos falso positivos (Sandin, Chorot, Valiente, Lostao $\&$ Santed, 2002). El hecho de que el impacto emocional disminuya luego de descartar el diagnóstico también confirma la importancia de esta sugerencia.

Sandin et al. (2002) compararon el impacto psicológico entre las pacientes llamadas para realizar una segunda prueba de screening y aquéllas en exámenes de rutina, encontrando un mayor nivel de preocupación, temor y creencia de padecer cáncer en el primer grupo. Estas tres variables estaban también correlacionadas con los niveles de ansiedad y depresión en los dos grupos. Otros estudios encontraron que el impacto psicológico negativo al ser llamadas para posteriores exámenes confirmatorios parece disminuir luego de recibir resultados finales negativos (Lampic et al., 2001). De todas maneras, Lowe y Balanda (1999) encontraron que el distrés en estas mujeres no disminuía luego de un mes, aun cuando el diagnóstico de cáncer fuera finalmente descartado.

La reducción del impacto psicológico a lo largo del tiempo también determina una declinación en la adherencia a programas de screening. Lipkus et al. (2000) compararon este comportamiento entre mujeres que habían recibido mamografías patológicas en los dos años previos y aquellas que las habían recibido antes de ese periodo (todas siendo finalmente resultados falso-positivos), encontrando que las mujeres en el primer grupo eran más proclives a realizar mamografías posteriores. Esto sugiere que el temor al cáncer es mayor en el primer periodo y de esa manera determina la adhesión a programas de screening. Sería interesante estudiar si las mujeres que cumplen estos programas también mantienen la adherencia a los tratamientos cuando un diagnóstico positivo de cáncer es finalmente detectado.

Se sabe ya que los proveedores de salud juegan un importante rol en motivar a las mujeres de bajos ingresos a realizarse estos exámenes. Estos comportamientos saludables pueden promoverse a través de breves intervenciones centradas en habilidades de afrontamiento relacionadas con el distres específico frente al cáncer. Audrain et al. (1999) encontraron que luego de este tipo de intervenciones las mujeres con mayor nivel de distrés mostraban una mejoría en su adherencia al auto-examen en comparación con las mujeres con bajo distrés. De esta manera, el primer grupo pareció beneficiarse tanto de esta intervención específica como de un programa de promoción desalud. Un hallazgo interesantees quela variable determinante no fue el riesgo percibido a padecer cáncer sino el malestar psicológico asociado a este estudio.

En la revisión de Hay, Buckley y Ostroff (2005) encuentran que, de acuerdo a los modelos fundados en la conducta de salud, la preocupación por tener cáncer puede facilitar o inhibir la adherencia a los programas de screening y esto nos habla de modos de 
afrontamientos antagónicos frente al mismo estresor.

Brett, Bankhead, Henderson, Watson y Austoker (2005) destacan queno es la mamografía en sí misma la que provoca efectos psicológicos adversos sino que el aumento del monto de ansiedad se asocia con el segundo Ilamado. Identifica también los factores asociados en los estudios con el impacto psicológico adverso y éstos son menor nivel educativo, vivir en zonas urbanas, tener un hijo o no y factores relacionados con el propio screening, talescomo insatisfacción con la información brindada, la demora entre una y otra instancia, el dolor durante los procedimientos y la preocupación por tener cáncer.

Dela revisión realizada surgela importancia de evaluar las respuestas de las personas frente a situaciones potencialmente estresantes como podría serlo el llamado a completar estudios de screening.

La evaluación de un estímulo como amenazante dispara el proceso de afrontamiento, y esto es claro frente al Ilamado a completar los estudios ya que enfrenta a las mujeres con la posibilidad de tener cáncer. Los mecanismos deafrontamiento están relacionados con la forma en que se evalúa la situación y el esfuerzo por superar la demanda. Lazarus define el afrontamiento como "permanentes esfuerzos cognitivos y comportamentales para manejar demandas específicas, externas o internas, que son evaluadas como excediendo los recursos de la persona" (Lazarus, 1993, p. 237). El concepto subraya el intento de reducir una demanda externa o interna a través de un esfuerzo intrapsíquico (cognitivo 0 emocional) o una acción.

El presentetrabajo pretende conocer cómo afrontan las mujeres esta amenaza (segundo llamado) y la consiguiente respuesta emocional. Se establecerá la relación entre la clasificación mamográfica como evidencia objetiva dela probabilidad tener cáncer y los mecanismos de afrontamiento así como la asociación entre estos mecanismos y la existencia deantecedentesfamiliares de cáncer demama.
Secompararán el mecanismo de afrontamiento de acuerdo al diagnóstico final (cáncer 0 no cáncer) y se buscará establecer predictores de cuadros psicopatológicos frecuentes (depresión y ansiedad).

La hipótesis de trabajo es que en aquel grupo de pacientes llamadas para realizar una segunda mamografía y/ o completar estudios se produce un impacto psicológico negativo, similar a otras situaciones de estrés agudo.

\section{MATERIALESY METODOS}

Setrata deun estudio descriptivo observacional utilizando métodos cualitativos y cuantitativos.

La población estudiada correspondea pacientes derivadas que concurren por primera vez a la Unidad de M astología del Hospital Universitario de M ontevideo derivada de programas de screening o por médicos de familia, las que consultaron por diferentes síntomas tales como dolor de mamas, nódulos o mastitis, previa realización de estudios entre julio de 2000 y noviembre de 2002.

Fueron estudiadas 377 pacientes, las que representaron el $84 \%$ de las primeras consultas, $2 \%$ rechazo participar, las otras no pudieron completarse debido al alto flujo de la consulta. La mayoría de ellas fueron derivadas allí luego de estudios de rutina (mamografías) para completar su examen. Los resultados mamográficos fueron clasificados de acuerdo al Breast Imaging Reporting and Data System (BI-RADS). Antes del examen médico las pacientes fueron entrevistadas y se les administró dos cuestionarios: la Escala de Ansiedad y Depresión Hospitalarias (HADS) y la escala Perfil de estados deánimo (POMS) para evaluar síntomas depresivos y ansiosos además de otros síntomas prevalentes. Se solicitó su consentimiento informado. Las entrevistas protocolizadas estuvieron centradas en sus reacciones frente a esta etapa prediagnósticay la forma deafrontamiento frente a la misma. 
El promedio de duración de las entrevistas fue de 9 minutos. Fueron grabadas y analizadas deacuerdo al $\mathrm{M}$ anual de $\mathrm{M}$ ecanismos deAfrontamiento deUIm. Tres entrevistadores entrenados puntuaron las entrevistas. LoS evaluadores no conocían el diagnóstico.

\section{Medidas \\ Clasificación mamográfica}
BI-RADS
1. Mamografía negativa.
2. Hallazgos benignos.
3. Probables hallazgos benignos.
4. Significativa probabilidad de cáncer.
5. Alta probabilidad de cáncer.

\section{Manual de MecanismosdeAfrontamiento de}

UIm: El instrumento es la entrevista semi-estructurada que es evaluada de acuerdo al manual. El mismo provee un método cualitativo (análisis del contenido de entrevistas semi-estructuradas) para evaluar la forma en quelas pacientes enfrentan el estrés. Esta técnica fue desarrollada en UIm y pareceser más útil para la investigación que los cuestionarios. Se basa en la definición de 35 diferentes estrategias de afrontamiento. El manual sugiereagruparlas en clusters: Resignación, Distracción, Reestructuración Cognitiva, Adhesión al Tratamiento, Espíritu de lucha y Contacto Social.

Escala HADS: Escala de Ansiedad y Depresión H ospitalarias (Snaith \& Zigmond, 1994). Esta escala fue diseñada para usarse con pacientes médicos y quirúrgicos. Mide niveles de ansiedad y depresión brindando resultados independientes para los mismos. Consiste en 14 ítemes. La interpretación de los re sultados es la siguiente: 0-7, normal; 8-10, depresión o ansiedad leve; 11-14 moderada; 15-21 severa.

Escala POM S: Perfil deestados deánimo (MC Nair-Douglas, 1992). Esta escala es ampliamenteutilizada y está estandarizada para eva- luar el estado afectivo. Los resultadosaltosindican una gran alteración emocional. Mide la alteración emocional general, la tensión ansiosa, la confusión, la depresión y tristeza, ira y hostilidad, fatiga y vigor.

\section{Análisis estadístico}

Serealizó un análisis descriptivo delos datos. Para evaluar las diferencias entre los grupos se utilizaron tests paramétricos (test de $t$ ) 0 no paramétricos ( $M$ ann Whitney) en función dela distribución de los datos.

Se realizó el análisis multivariado de los predictores de depresión y ansiedad a través de la regresión múltiple stepwise, tomando como variable dependiente los puntajes continuos del HADS.

\section{RESULTAD OS}

Un total de 377 pacientes fueron entrevistadas y testadas. La media de edad fue 48 años, mediana 47 (R: 25-84, SD: 11.22).

Luego de analizar las entrevistas fueron detectados un total de 996 eventos de afrontamiento, lo que resultó en un promedio de 2.8 por entrevista. Treinta y siete pacientes ( 10 \%) fueron diagnosticadas con cáncer de mama. Estas pacientes reportaron 125 de los eventos de afrontamiento (Me:4, R:1-13), mientras quelas pacientesque no tenían cáncer reportaron 871 (Me:2, R:0-9), siendo la diferencia estadísticamente significativa (M ann-Whitney test - two tailed: $p<0.0001$ ).

La escala HADS mostró niveles moderados de ansiedad y depresión en ambos grupos, sin diferencias estadísticamente significativas $(P \geq 0,5)$.

Las pacientes también fueron comparadas de acuerdo a la evidencia presentada (clasificación mamográfica), utilizando los clusters de afrontamiento del manual.

Las pacientesfueron agrupadas en tresgrupos: $\mathrm{G} 1$ ( $\mathrm{n}: 279$ ) para BI-RADS 1, 2 y 3; G2 (n :48) para aquellas con presencia de síntomas 
clínicos y G3 (n:30) para BI-RADS 4 y 5. Al estudiar las estrategias de afrontamiento de acuerdo a los resultados mamográficos y síntomas clínicos, las pacientes $\mathrm{G} 3$ mostraron un mayor uso del cluster resignación, como modo de afrontamiento $(p<0.001)$ y un me nor uso del cluster aceptación ( $p<0.003)$.

Se dividieron y analizaron los grupos teniendo en cuenta los antecedentes familiares de cáncer de mama. La percepción de riesgo debido al conocimiento de estos antecedentes no determinó diferencias significativas entre los grupos.

Al estudiar los mecanismos de afrontamiento en relación al diagnóstico final, los mecanismos pertenecientes al cluster resignación fueron utilizados por el $49 \%$ de las pacientes que finalmente recibieron un diagnóstico positivo, comparado con el $30 \%$ de las sanas $(p<0.04)$. El $51 \%$ de las pacientes sin diagnóstico de cáncer utilizaron los mecanismos del cluster aceptación, mientras quesólo el $24 \%$ de las pacientes con cáncer lo hicieron $(p<0.003)$.

Al realizarse el análisis de los mecanismos de afrontamiento por persona sin tener en cuenta el número de eventos, la aceptación activa fue utilizada por el $45 \%$ de las pacientes sin cáncer ( $N$ : 151, p<0.05). Los mecanismos de afrontamiento utilizados por las pacientes con cáncer fueron la evitación activa ( $\mathrm{N}: 12,32 \%, \mathrm{p}<0.05)$ y la desesperanza (17 $\%, p<0,01)$.

Si tomamos el total de los 35 mecanismos de afrontamiento sin agruparlos por clusters , encontramos que la estrategia más frecuentemente usada por las pacientes sanas fue la aceptación activa y esto representó el $20 \%$ del total de los eventos de afrontamiento, mientras que la estrategia más usada por las pacientes con cáncer fue la evitación activa (11\%).

En relación a los predictores de cuadros psicopatológicos, la regresión lineal múltiple para predictores de depresión incluidos en el estudio explicaron el $54 \%$ de la varianza. Los predictores más frecuentes fueron la depre- sión, el bajo vigor y actividad (de acuerdo al POMS) y el mayor uso de la desesperanza como mecanismo deafrontamiento. La regre sión lineal múltiple (Stepwise) con la ansiedad (HADS) como variable dependientemostró un modelo explicando el $50 \%$ de la varianza. El modelo incluyó mayores niveles de tensión ansiosa, confusión (POM S), desesperanza y cavilación como mecanismos de afrontamiento (Manual de UIm).

\section{DISCUSION Y CONCLUSIONES}

En primer lugar debedestacarse quetodas las mujeresincluidas en esteestudio consultaron por propia iniciativa, en la mayoría de los casos por prevención. Este hecho seguramente marca un perfil diferencial con aquellas pacientes que consultan tardíamente con la enfermedad constituida.

La presentación de los resultados se realiza de acuerdo al diagnóstico final (presencia 0 ausencia de cáncer). Seobserva quela aceptación activa fuela estrategia de afrontamiento más utilizada por las mujeres con patología benigna o sin patología, que se acompañó de una actitud activa y cuidadosa hacia su salud, concurriendo inmediatamente al segundo llamado a pesar de una muy baja probabilidad de padecer cáncer. Contrariamente, las mujeres quefinal menterecibieron el diagnóstico de cáncer, demoraron en la segunda consulta. La mayor probabilidad de tener cáncer de acuerdo a la clasificación de la mamografía generó respuestas pasivas de desesperanza y de evitación de la consulta. Estas mujeres utilizaron más frecuentemente un patrón de resignación como respuesta a la amenaza y una evitación activa de la consulta, lo que se manifestó por una consulta tardía luego del segundo llamado.

Al anal izar la respuesta emocional (POM S, HADS) no se encontraron diferencias significativas entre los grupos ( cáncer-no cáncer), así como tampoco entre aquellas mujeres que tenían antecedentes familiares de cáncer de 
mama y quienes no lo tenían. Sin embargo, la presencia de niveles moderados de ansiedad en la muestra indicó que la situación específica de la realización de la mamografía de screening es estresante en sí misma. El hecho de ser derivada a un centro oncológico parece ser más amenazante que el resultado específico de la mamografía (clasificación Birads). La sola presencia de mecanismos de afrontamiento, de acuerdo a la definición de los mismos, es un indicador de que la situación esuna exigencia parala persona. Losmecanismos de afrontamiento permitieron observar diferencias entre los grupos más claramente que los estados emocionales. Las mujeres finalmente diagnosticadas con cáncer presentaron un mayor número de eventos de afrontamiento en relación a las sanas, siendo esta diferencia estadísticamente significativa. En este grupo se encontró asociación entre la presencia de ansiedad como estado emocional, medido por el POM Sy la desesperanza y cavilación como mecanismos deafrontamiento. La desesperanza, aunque es un mecanismo de afrontamiento, nos habla de la capitulación de los esfuerzos, ya quela personasiente que nada puede cambiar o hacer. La cavilación se relaciona con un tipo de pensamiento permanente, estresante, mortificante y compulsivo acerca del problema, sin poder realizar ninguna acción para resolverlo. La desesperanza y la cavilación son tipos de afrontamiento de alto riesgo ya que inhiben la consulta, por lo queel personal desalud debeformarseen su precoz identificación. Asimismo, losniveles moderados dedepresión en el grupo de pacientes también correlacionaron con la desesperanza como mecanismo de afrontamiento y la falta de vigor y actividad frente a la amenaza.

El temor al cáncer genera, en el grupo de mayor riesgo, una conducta evitativa y dedesesperanza en la etapa diagnóstica. El posible diagnóstico de cáncer pareceinmovilizar a las pacientes al comienzo de la enfermedad, lo que no significa que el subsiguiente proceso se caracterice por ello. Se plantea entonces la interrogante acerca de la estabilidad de los mecanismos de afrontamiento, es decir si los mecanismosquepresenta una pacienteen ese momento representan su estilo habitual o sólo se activan frente a determinadas situaciones estresantes. La pregunta sería entonces si el afrontamiento es un rasgo estable o un estado transitorio.

En la población estudiada observamos que las estrategias evitativas y de desesperanza se incrementan frente a la fuerte evidencia de tener cáncer y su diagnóstico. Aunque el número de pacientes con cáncer en la muestra es pequeño, los resultados muestran una tendencia que es necesario confirmar en otros estudios. Las metodologías cualitativas utilizadas son costosas en tiempo y requieren de personal especializado, por lo que se hace necesario, a partir de los datos de este estudio, seleccionar escalas que permitan identificar precozmentelosmecanismos de afrontamiento de riesgo para las pacientes.

La inhibición delas conductas deautocuidado representa el principal grupo de riesgo a la hora de planificar e implementar intervenciones. La detección delos mecanismos de afrontamiento permitedecidir la intervención psicosocial eimplementar estrategias de evaluación de resultados, a través de la evaluación de la modificación o persistencia de los patronesidentificados. La intervención apuntaría a brindar soporte emocional y a la transmisión de información, haciendo especial énfasis en la posibilidad de estar frente a resultados falso-positivos por un lado, a la vez queinformando sobrela importancia del tratamiento precoz en caso de confirmar el diagnóstico.

\section{REFERENCIAS}

Absetz, P., Aro, A.R., Sutton, S.R. (2003). Experience with breast cancer, pre-screening perceived susceptibility and the psychological impact of screening. Psycho-Oncology, 12: 305318.

Audrian, J., Rimer, B., Cella, D., Stefanek, M., 
Garber, J., Pennanen, M . et al . (1999) . The impact of a brief coping skills intervention on adherence to breast self-examination among first-degreerelatives of newly diagnosed breast cancer patients. Psycho-O ncology, 8, 220-229.

Banks, E., Beral, V., Cameron, R., Hogg, A., Langley, N., Barnes, I. et al. 2002. Comparison of various characteristics of women who do and do not attend for breast cancer screening. Breast Cancer Res. Recuperado de: http:// breast-cancer-research.com/ content/ 4/1/R1

Bjurstam, N ., Björneld, L., Warwick, J. (2003). The Gothenburg Breast Screening Trial. Cancer, 10: 2387-96.

Blanchard, K., Colbert, J., Puri, D., Weissman, J., M oy, B., Kopans, D. et al . (2004). M ammography screening: Patterns of use and estimated impact on breast carcinoma survival. Cancer, 101, 495-507.

Brett, C., Bankhead, B., Henderson, B., Watson, E., Austoker, J. (2005). The psychological impact of mammographic screening. A systematic review. Psycho-O ncology, 14, 917-938.

Buseman S. et al. (2003). M ammography screening matters for young women with breast carcinoma, evidence of down staging among 4249-year-old women with a history of previous mammography screening. Cancer, 97, 352-358.

Duffy, S.W., Tabár, L. \& Chen, H . (2002). The impact of organized mammography service screening on breast carcinoma mortality in seven Swedish countries. Cancer, 95, 458-469.

Hay, J., Buckley, T. \& Ostroff, J., (2005) The role of cancer worry in cancer. Screening: a theoretical and empirical. Psycho-O ncology, 14, 517534 DOI: 10.1002/pon.864

Lampic, C., Thurfjell, E., Bergh, J. \& Sjödén, P.O. (2001). Short- and long-term anxiety and de- pression in women recalled after breast cancer screening. European Journal of Cancer, 37, 463469.

Lazarus, R. (1993). Coping theory and research: past, present, and future. Psychosom. M ed., 55, 234-247).

Lipkus, I.M ., Halabi, S., Strigo, T.S. \& Rimer, B.K. (2000). The impact of abnormal mammograms in psychosocial outcomes and subsequent screening. Psycho-O ncology, 9, 402-410.

Lowe, J.B. \& Balanda, K.P. (1999). Psychologic distressin women with abnormal findingsin mass mammography screening. Cancer, 85, 11141118.

McNair-Douglas, M . (1999). POM SM anual. EDITS.

M ichaelson, J.S., Satija, S., Kopans, D., M oore, R., Silverstein, M.,Comegno, A. et al. (2003). Gauging the impact of breast carcinoma screening in terms of tumour size and death rate. Cancer, 98, 2114-2124

Sandin, B., Chorot, P., Valiente, R.M ., Lostao, L. \& Santed, M .A. (2002). Adversepsychological effects in women attending a second-stage breast cancer screening. Journal of Psychosomatic Research, 52, 303-309.

Stratman, H., André, L., Verbeek, M., Petronella, G., Peer, M . \& Borm, G. (2004). Estimating life expectancy and related probabilities in screendetected breast cancer patients with restricted follow-up information. Statistics in M edicine, 23, 431-448.

Snaith, R.P \& Zigmond, A.S. (1994). The hospital anxiety and depression scale. M anual.

Tabár, L. 2001. Organized mammography screening substantially reduces breast carcinoma mortality. Cancer, 91, 1724-31. 